\title{
Nonlinear Spectroscopic Effects in Quantum Gases Induced by Atom-Atom Interactions
}

\author{
A. I. Safonov ${ }^{a, b}$ * I. I. Safonova ${ }^{a}$, and I. S. Yasnikov ${ }^{c}$ \\ ${ }^{a}$ National Research Centre Kurchatov Institute, 123182 Moscow, Russia \\ ${ }^{b}$ Moscow Institute of Physics and Technology (State University), 141700 Dolgoprudny, Moscow region, Russia \\ ${ }^{c}$ Togliatti State University, 445667 Togliatti, Russia
}

(Dated: August 28, 2012; in final form, October 23, 2012)

\begin{abstract}
We consider nonlinear spectroscopic effects - interaction-enhanced double resonance and spectrum instability - that appear in ultracold quantum gases owing to collisional frequency shift of atomic transitions and, consequently, due to the dependence of the frequencies on the population of various internal states of the particles. Special emphasis is put to two simplest cases, (a) the gas of twolevel atoms and (b) double resonance in a gas of three-level bosons, in which the probe transition frequency remains constant.
\end{abstract}

\section{INTRODUCTION}

As is well-known, interaction of a multilevel quantum system simultaneously with several resonance fields is, under certain conditions, accompanied by nonlinear spectroscopic phenomena, coherent population trapping (CPN) 1] and electromagnetically induced transparency (EIT) 2], which are caused by the formation of a special "dark" superposition state immune to the resonance fields. These effects, however, are still linear in a sense that the resonance transition frequencies in the quantum system are independent of the population of various states. In this work, we consider another, generally speaking, a more general class of phenomena induced by exactly such a dependence exemplified by collisional or contact frequency shift of intra-atomic (e.g., hyperfine) transitions in quantum gases owing to the interaction between of the gas particles. In our previous work [3] , we showed that interaction of a gas of three-level atoms with two light fields results, due to the contact shift, in a specific kind of double resonance, interaction-enhanced double resonance (INEDOR). In addition, as will be shown below, a gas of two-level atoms with a nonzero contact shift can exhibit spectrum instability of the resonance transition, namely, the dependence of the resonance line shape and the final population of the levels on sweep direction, as well as on the relation between the amplitude of the probe field, sweep rate and the magnitude of the contact shift.

The behavior of an arbitrary two-level system is commonly described in terms of effective spin (pseudo-spin) $s=1 / 2$. We will also follow this representation, in each case attributing spin to a particular pair of quantum states coupled by a resonance transition. In this work, we disregard spin waves and related effects associated with spatial transport of spin polarization, which actually corresponds to zero spin diffusion constant. Below we will discuss to what extent this assumption corresponds to

*Electronic address: alesaf2008@rambler.ru real physical systems. Spatial inhomogeneity is included only in the analysis of a possible INEDOR spectrum line shape in a linear gradient of the external field.

\section{NONLINEAR DYNAMICS OF A THREE-LEVEL SYSTEM}

In general, the evolution of a quantum system interacting with resonance fields is described by Liouville-von Neuman equation for the components of the spin density matrix $\rho$ [4]

$$
i \hbar \frac{\partial \rho}{\partial t}=[\hat{H}(t), \rho]-i \hat{\mathcal{L}}(t) \rho,
$$

where $\hat{H}(t)=\hat{H}_{0}+\hat{U}(t)$ is the Hamiltonian including the unperturbed term $\hat{H}_{0}$ and the time-dependent perturbation $\hat{U}(t)$ due to the external ac field, $\hat{\mathcal{L}}$ is the Lindblad superoperator [5] responsible for dissipation and square brackets, as usually, denote quantum-mechanical commutation.

To obtain the general evolution equation for the components of the density matrix of a gas of three-level atoms interacting with two resonance fields we use the previously derived relation for the contact shift of the transition $|1\rangle \rightarrow|2\rangle$ in a spatially homogeneous gas in the presence of the third state $|3\rangle[\underline{6}$. We will be interested in coherent population of the states. In this case, the frequency shift $\Delta_{i j}(\rho) \equiv \omega_{i j}(\rho)-\omega_{i j}(0)(i, j=1,2,3)$ of the transition between the states $|i\rangle$ and $|j\rangle$ at a certain nonzero gas density $n \equiv \operatorname{Tr}\left(\rho_{i j}\right)$ vanishes for fermions, whereas for bosons in the absence of a Bose-Einstein condensate it is

$$
\begin{aligned}
\hbar \Delta_{12} & =\left(\rho_{11}+\rho_{22}\right) \delta \lambda_{12}+ \\
& +\left(\rho_{22}-\rho_{11}\right) \Delta \lambda_{12}+2 \rho_{33}\left(\lambda_{23}^{+}-\lambda_{13}^{+}\right) \\
\hbar \Delta_{13} & =\left(\rho_{11}+\rho_{33}\right) \delta \lambda_{13}+ \\
& +\left(\rho_{33}-\rho_{11}\right) \Delta \lambda_{13}+2 \rho_{22}\left(\lambda_{23}^{+}-\lambda_{12}^{+}\right) .
\end{aligned}
$$

Here, $\rho_{i j}$ are the components of the density matrix in terms of the eigen wavefunctions of the unperturbed Hamiltonian $\hat{H}_{0}, \delta \lambda_{i j}=\lambda_{j j}-\lambda_{i i}, \Delta \lambda_{i j}=\lambda_{i i}+\lambda_{j j}-2 \lambda_{i j}^{+}$, 


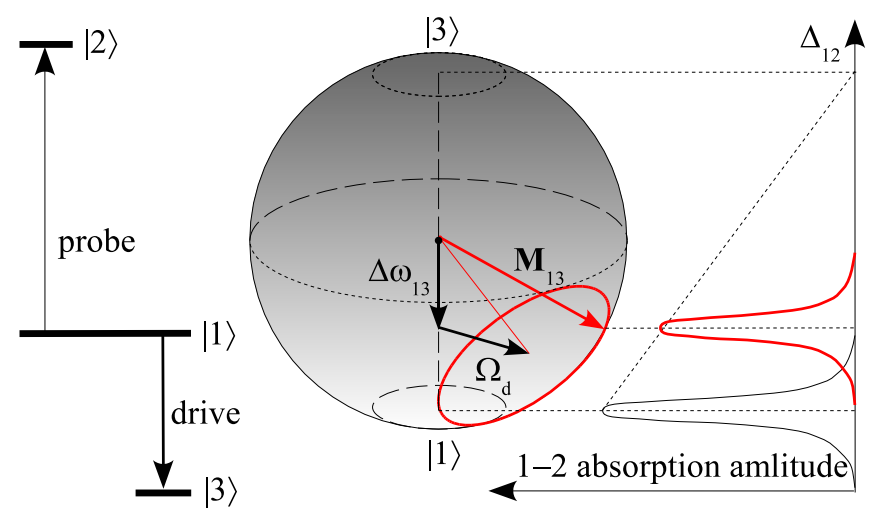

Figure 1: Scheme of (left) a three-level system and (right) influence of the Rabi oscillations between the states $|1\rangle$ and $|3\rangle$ on the $|1\rangle-|2\rangle$ transition frequency and intensity. For clarity, the Rabi period $2 \pi \Omega_{\mathrm{d}}^{-1}$ of the drive transition is assumed to be much longer than the detection time of the $|1\rangle-|2\rangle$ resonance line.

$\lambda_{i j}^{ \pm} \equiv\langle i j|\lambda| i j\rangle_{ \pm}$is the spin part of the matrix element of the interaction intensity $\lambda=4 \pi \hbar^{2} a / m$, which is commonly used to describe cold collisions, when the partial amplitudes of scattering with a nonzero angular momentum of the relative motion of the colliding particles "freeze out", $m$ is the atomic mass, $a$ is the respective s-wave scattering length. The superscript "+" denotes that the wavefunction of two colliding bosons is symmetric with respect to permutation of both their pseudo-spin and spatial coordinates. The doubly antisymmetric component, obviously, do not contribute to s-wave scattering.

Below we restrict ourselves to the typical situation in double-resonance experiments, when the state $|2\rangle$ is initially unpopulated and its population changes insignificantly during the interaction with a weak probe field with the frequency $\omega_{\mathrm{p}}$, whereas the populations of the states $|1\rangle$ and $|3\rangle$ can vary quite arbitrarily under the action of a strong drive field with the frequency $\omega_{\mathrm{d}}$. In this case, $\rho_{22} \ll \rho_{11}, \rho_{33}$, and therefore the last term in the righthand side of Eq. (2.3) can be omitted. Thus, the general equation (2.1) for the evolution of the components of the density matrix of the gas of three-level bosons with a ladder $(\Xi)$ level scheme (Fig. (1) in the absence of spontaneous longitudinal relaxation becomes (cp. 1])

$$
\begin{aligned}
i \frac{\partial \rho_{11}}{\partial t} & =\frac{\Omega_{\mathrm{d}}}{2} \rho_{13} \exp \left[i \Delta \omega_{13}(\rho) t\right]- \\
& -\frac{\Omega_{\mathrm{p}}}{2} \rho_{21} \exp \left[i \Delta \omega_{12}(\rho) t\right]-\text { c.c., } \\
i \frac{\partial \rho_{33}}{\partial t} & =-\frac{\Omega_{\mathrm{d}}}{2} \rho_{13} \exp \left[i \Delta \omega_{13}(\rho) t\right]+\text { c.c. } \\
i \frac{\partial \rho_{31}}{\partial t} & =\frac{\Omega_{\mathrm{d}}}{2}\left(\rho_{33}-\rho_{11}\right) \exp \left[i \Delta \omega_{13}(\rho) t\right]+ \\
& +\frac{\Omega_{\mathrm{p}}}{2} \rho_{32} \exp \left[i \Delta \omega_{12}(\rho) t\right]-i \gamma_{13} \rho_{31}, \\
i \frac{\partial \rho_{22}}{\partial t} & =\frac{\Omega_{\mathrm{p}}}{2} \rho_{21} \exp \left[i \Delta \omega_{12}(\rho) t\right]-\text { c.c. }
\end{aligned}
$$

$$
\begin{aligned}
i \frac{\partial \rho_{12}}{\partial t} & =\frac{\Omega_{\mathrm{p}}}{2}\left(\rho_{22}-\rho_{11}\right) \exp \left[i \Delta \omega_{12}(\rho) t\right]+ \\
& +\frac{\Omega_{\mathrm{d}}}{2} \rho_{32} \exp \left[i \Delta \omega_{13}(\rho) t\right]-i \gamma_{12} \rho_{12}, \\
i \frac{\partial \rho_{32}}{\partial t} & =-\frac{\Omega_{\mathrm{d}}}{2} \rho_{12} \exp \left[i \Delta \omega_{13}(\rho) t\right]- \\
& -\frac{\Omega_{\mathrm{p}}}{2} \rho_{31} \exp \left[i \Delta \omega_{12}(\rho) t\right]-i \gamma_{32} \rho_{32},
\end{aligned}
$$

where $\Omega_{\mathrm{p}(\mathrm{d})}$ and $\Delta \omega_{12(13)}(\rho) \equiv \omega_{\mathrm{p}(\mathrm{d})}-\omega_{12(13)}(\rho)$ are the Rabi frequency and density-dependent frequency detuning of the probe (drive) field and $\gamma_{i j}$ are the transverse relaxation rates. The light fields are thought to be spatially homogeneous, which usually holds for hyperfine transitions, whose wavelengths are much larger that the geometrical size of the sample.

If, as already mentioned above, the probe field is much weaker than the drive field, $\Omega_{\mathrm{p}} \ll \Omega_{\mathrm{d}}$, the second term in Eqs. (2.4) and (2.6) can be neglected. In this case, Eqs. (2.4)-(2.6) can be written in a more compact form of a usual Bloch equation for the precession of the spin polarization ("magnetization") vector $\mathbf{M}$ in the Hilbert space of the states $|1\rangle$ and $|3\rangle\left(\mathbf{M}_{z}=\left(0,0, \rho_{33}-\rho_{11}\right)\right.$, $\left.\mathbf{M}_{\perp}=2\left(\operatorname{Re} \rho_{13}, \operatorname{Im} \rho_{13}, 0\right)\right)$ :

$$
\frac{\partial \mathbf{M}}{\partial t}=\mathbf{M} \times\left(\tilde{\boldsymbol{\Omega}}+\frac{\Delta \lambda_{13}}{\hbar} \mathbf{M}_{z}\right)-\gamma_{13} \mathbf{M}_{\perp}
$$

where $\tilde{\boldsymbol{\Omega}}=\left(\Omega_{\mathrm{d}}, 0, \omega_{13}(0)-\omega_{\mathrm{d}}+\hbar^{-1} \delta \lambda_{13}\left(\rho_{11}+\rho_{33}\right)\right)$. For clarity, we separate in the precession frequency $\tilde{\boldsymbol{\Omega}}$ the component that remains nearly constant in a weak probe field, when $\rho_{11}+\rho_{33} \approx$ const. The second term in the parenthesis in the right-hand side of Eq. (2.10) expresses explicitly the dependence of the precession frequency on the current value of the magnetization vector $\mathbf{M}$. In the subsequent sections, we discuss the direct consequences of this, generally speaking, nonlinear precession in two limiting cases.

\section{INTERACTION-ENHANCED DOUBLE RESONANCE}

As follows from Eq. (2.2), excitation of the transition from the state $|1\rangle$ to $|3\rangle$ in a Bose gas induces frequency modulation of the transition $|1\rangle-|2\rangle$ associated with the Rabi oscillations of the populations of the states $|1\rangle$ and $|3\rangle$ [6]. If both transitions are excited simultaneously, this modulation leads to a new phenomenon, interactionenhanced double resonance.

Here, for simplicity and clarity, we restrict ourselves to the case $\lambda_{11}=\lambda_{12}^{+}$, when the contact shift of the transition $|1\rangle-|2\rangle$ vanishes at $\rho_{22}=\rho_{33}=0$. Another advantage of this case is that it allows direct comparison with the experiments on electron-nuclear double resonance (ENDOR) in atomic hydrogen [9, 13]. To simplify our consideration even further, we assume that the frequency of the drive transition $|1\rangle-|3\rangle$ remains constant, which requires $\Delta \lambda_{13}$ to be sufficiently small. An 
opposite case is considered in Sec. IV] Despite the above assumptions, a general analytic solution of nonlinear system (2.7)-(2.10) is hardly possible. The approach implemented below does not feature such generality but provides quantitative and physically transparent description in the case of interest.

Physics of the novel kind of double resonance becomes clear from Fig. 1, In the absence of relaxation, the populations of the states $|1\rangle$ and $|3\rangle$ oscillate under continuous excitation of the transition $|1\rangle-|3\rangle$ in the initially pure state $|1\rangle$ sample at the frequency (see Fig. 1) $\tilde{\Omega}_{13}=\sqrt{\Omega_{\mathrm{d}}^{2}+\Delta \omega_{13}^{2}}$. This results in the frequency modulation of the probe transition $|1\rangle-|2\rangle[\underline{6}]$

$$
\hbar \omega_{12}=\hbar \omega_{12}(0)+2 n \Delta \lambda\left(\frac{\Omega_{\mathrm{d}}}{\tilde{\Omega}_{13}}\right)^{2} \sin ^{2}\left(\frac{\tilde{\Omega}_{13} t}{2}\right),
$$

where $\Delta \lambda=\lambda_{23}^{+}-\lambda_{13}^{+}$. The amplitude of this frequency modulation can easily be comparable with or even much greater than the linewidth of the probe transition. In this case, excitation of the transition $|1\rangle-|3\rangle$ periodically drives the probe transition out of resonance. For clarity, Fig. 1 corresponds to slow driving in a sense that the Rabi period $2 \pi \Omega_{\mathrm{d}}^{-1}$ of the drive transition is thought to be much longer than the time needed to detect the $|1\rangle-|2\rangle$ resonance line and the entire $|1\rangle-|2\rangle$ spectrum moves periodically fore and back along the frequency axis and simultaneously changes in amplitude. The waveforms shown in Fig. 1 are the schematic "snapshots" of the spectrum at different phases of the Rabi cycle. Obviously, electromagnetic absorption at the fixed frequency $\omega_{\mathrm{p}}$ of the probe field also changes periodically with a period of the $|1\rangle-|3\rangle$ Rabi oscillations. It should be emphasized that these changes are associated with changes in both the population of the initial state and the transition frequency, in contrast to conventional double resonance, which is solely due to a change in the population of the initial state caused by the drive transitions. Clearly, such interaction-induced frequency modulation can greatly enhance the effect, which therefore can be called INteraction-Enhanced DOuble Resonance (INEDOR).

Let us consider in more detail a possible line shape of the INEDOR spectrum in a strong drive field when, in contrast to the case illustrated in Fig. 1. simultaneous frequency and amplitude modulation of the $|1\rangle-|2\rangle$ absorption line is relatively fast and therefore is integrated by the detection system. This implies that the $|1\rangle-|3\rangle$ Rabi frequency is much higher than the rate of field or frequency sweep through the $|1\rangle-|2\rangle$ resonance or the inverse time constant $\tau^{-1}$ of the detection system, $\Omega_{\mathrm{d}} \tau \gg 1$.

Generally, the energies of all three levels and, consequently, both transition frequencies depend on the external static field. The specific nature of this field is insignificant but for definiteness we shall consider the magnetic field $B$. Let the field $B_{0}$ correspond to the exact resonance $|1\rangle-|3\rangle$ at a certain density $\rho, \omega_{13}\left(\rho, B_{0}\right)=\omega_{\mathrm{d}}$.
Then the deviation $b=B-B_{0}$ of the field from this value is equivalent to the frequency detuning $\Delta \omega_{13}(\rho, b)=\gamma_{\mathrm{d}} b$ and $\Delta \omega_{12}(\rho, b)=\gamma_{\mathrm{p}} b$ of the drive and probe field, respectively (here, $\gamma_{\mathrm{d}(\mathrm{p})}$ is the corresponding gyromagnetic ratio). Owing to this Zeeman contribution to the frequency $\tilde{\Omega}_{13}(\rho, b)=\gamma_{\mathrm{d}} \sqrt{B_{\mathrm{d}}^{2}+b^{2}}$ of the magnetization precession forced by the drive field $B_{\mathrm{d}}(t)=B_{\mathrm{d}} \exp \left(i \omega_{\mathrm{d}} t\right)$, the amplitude of the oscillating component of $\omega_{12}$ in Eq. (3.1), i.e., the amplitude of the frequency modulation of the probe transition, is a Lorentzian function of the static field $b$. As a result, $\omega_{12}(t)$ oscillates between the Zeeman-only zero-density lower bound (dashed line in Fig. 2a a)

$$
\omega_{12}\left(0, B_{0}+b\right)=\omega_{12}\left(0, B_{0}\right)+\gamma_{\mathrm{p}} b
$$

and the upper bound, which is the sum of the Zeeman and contact-shift contributions (thick solid line in Fig. 2a),

$$
\omega_{12}\left(\rho, B_{0}+b\right)=\omega_{12}\left(0, B_{0}\right)+\gamma_{\mathrm{p}} b+\left(\frac{2 n \Delta \lambda}{\hbar}\right) \frac{B_{\mathrm{d}}^{2}}{B_{\mathrm{d}}^{2}+b^{2}},
$$

at the field-dependent frequency $\tilde{\Omega}_{13}(b)[3]$. According to Eq. (3.3), the upper bound of the probe frequency is generally a nonmonotonic function of the external static field.

The time-average absorption amplitude $A\left(b, \omega_{\mathrm{p}}\right)$ at the probe frequency within the bounds (3.2) and (3.3) is proportional to the average population of the initial state and the probability density to find the system at given values of $b$ and $\omega_{\mathrm{p}}$. The probability density, in turn, peaks at the lower and upper bound of the probe transition frequency, where the system spends more time, since in these cases, $d \omega_{12}\left(b, \omega_{\mathrm{p}}\right) / d t=0[\underline{6}]$.

To illustrate the effect of spatial inhomogeneity, let us consider in more detail an infinite sample with a spatially homogeneous density in a linear gradient $\nabla B$ of the static field and homogeneous light fields. In this case, different parts of the sample simultaneously experience all possible field values. The absorption amplitude at a given probe frequency $\omega_{\mathrm{p}}$ is proportional to the integral $I\left(\omega_{\mathrm{p}}\right)=\int A\left(b, \omega_{\mathrm{p}}\right)\left(\frac{\partial N}{\partial b}\right) d b$ along the line $\omega_{\mathrm{p}}=$ const within the segments $A B$ and $C D$ in Fig. 2 a. The result of numerical integration with the parameters of the ENDOR experiments with 2D atomic hydrogen at a density of $3 \cdot 10^{12} \mathrm{~cm}^{-2}$ in the strong polarizing magnetic field $B=45 \mathrm{kG}$ and the drive field $B_{\mathrm{d}}=1 \mathrm{mG}[9,13,14$, at the maximum amplitude $\Delta B_{\mathrm{c}}=2 n \Delta \lambda\left(\hbar \gamma_{\mathrm{p}}\right)^{-1}=89 \mathrm{G}$ of the contact shift of the hyperfine transition $|b\rangle-|c\rangle$ in field units (here, $\gamma_{\mathrm{p}}$ and $\gamma_{\mathrm{d}}$ are to a good accuracy equal to the gyromagnetic ratio of electron and proton, respectively), is shown in Fig. 2 $\mathrm{b}$ as a function of the drive frequency $\omega_{\mathrm{d}}$ (lower horizontal axis) at constant $\omega_{\mathrm{p}}=\omega_{12}\left(0, B_{0}\right)$. Alternatively, the INEDOR spectrum can be detected by sweeping $\omega_{\mathrm{p}}$ (upper horizontal axis) at constant $\omega_{\mathrm{d}}$ (in Fig. 2 $\mathrm{b}, \omega_{\mathrm{d}}=\omega_{13}\left(0, B_{0}\right)$ ). In this case, the spectrum is inverted on the frequency scale because an increase in $\omega_{\mathrm{d}}$ corresponds to an increase in the resonance value of the static field $\omega_{\mathrm{d}} / \gamma_{\mathrm{d}}$ and, consequently, to a positive displacement of the mean-field Lorentzian 

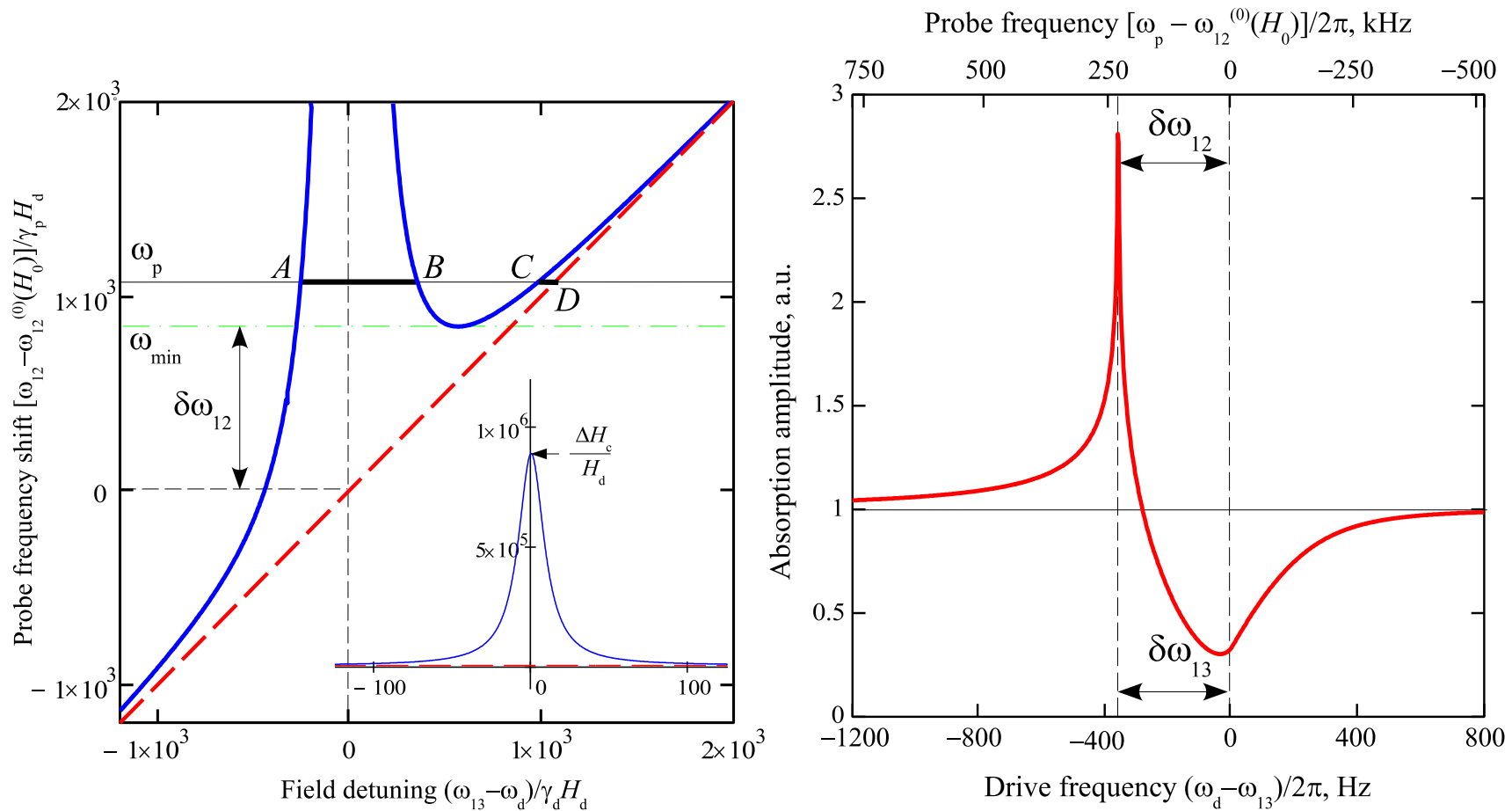

Figure 2: (a) Field dependence of the $|1\rangle-|2\rangle$ frequency shift (in units of $\gamma_{\mathrm{p}} H_{\mathrm{d}}$ ) under the CW excitation of the $|1\rangle-|3\rangle$ resonance. Horizontal axis is the field detuning $\left(\omega_{13}-\omega_{\mathrm{d}}\right) / \gamma_{\mathrm{d}}$ from the $|1\rangle-|3\rangle$ resonance in units of the excitation field $B_{\mathrm{d}}$. Solid and dashed line are, respectively, the upper bound of the sum of the Zeeman and mean-field contributions and the Zeeman contribution alone. Horizontal lines indicate (dash-dotted line) the value of $\omega_{12}$ at minimum and (solid line) the probe frequency. Vertical dashed line corresponds to the resonance field for the $|1\rangle-|3\rangle$ transition. Inset is the overview of the same dependence. (b) $|1\rangle-|2\rangle$ absorption amplitude as a function of the drive frequency (the lower horizontal axis is the detuning, in Hertz, from the $|1\rangle-|3\rangle$ resonance) for the monochromatic probe field with the fixed frequency $\omega_{\mathrm{p}}=\omega_{12}\left(0, B_{0}\right)$. Sharp peak corresponds to the minimum of the probe frequency in (a). The spectrum that appears when $\omega_{\mathrm{p}}$ (upper horizontal axis) is swept at fixed $\omega_{\mathrm{d}}=\omega_{13}$ is inverted with respect to the frequency axis. The parameters correspond to $2 \mathrm{D}$ atomic hydrogen with a density of $3 \cdot 10^{12} \mathrm{~cm}^{-2}$ in the high polarizing field $B=45 \mathrm{kG}$, except for the sign of the contact shift: $B_{\mathrm{d}}=1 \mathrm{mG}$, $\Delta B_{\mathrm{c}}=89 \mathrm{G}$.

peak (3.3) on the curve $\omega_{12}(b)$ in Fig. 2h. The latter is in turn equivalent to a decrease in the probe frequency $\omega_{\mathrm{p}}$ at constant $\omega_{\mathrm{d}}$. Which way of observing the INEDOR spectrum is preferred depends on the details of a particular experiment.

The absorption amplitude as a function of $\omega_{\mathrm{d}}$ (Fig. 2 2 ) decreases substantially within the drive resonance and has a sharp maximum at $\omega_{\mathrm{p}}=\omega_{\min }$, i.e., when the minimum value of Eq. (3.3) coincides with the probe frequency. This explains the dispersion-looking ENDOR spectra of 2D atomic hydrogen [9, 13]. Qualitatively, the hole in the absorption amplitude is because the atoms of the otherwise resonant regions of the sample are periodically driven out of the probe resonance and, as a result, spend only a small fraction of time in the resonance conditions. On the other hand, the absorption maximum is due to the fact that the drive resonance introduces zero gradient of the $|1\rangle-|2\rangle$ transition frequency in a certain region of the sample and, therefore, much more atoms become resonant.

The width of the double-resonance curve in the probefrequency units can be estimated as the difference $\delta \omega_{12}=$ $\omega_{\min }-\omega_{12}\left(0, B_{0}\right)$ (see Fig. $2 \mathrm{a}$ ). It is readily shown that for a sufficiently high amplitude of the contact shift such that $|2 n \Delta \lambda| \gg \hbar \gamma_{\mathrm{p}} B_{\mathrm{d}}$ and $b \gg B_{\mathrm{d}}[3]$,

$$
\delta \omega_{13}=\frac{\gamma_{\mathrm{d}}}{\gamma_{\mathrm{p}}} \delta \omega_{12} \simeq \frac{3}{2} \gamma_{\mathrm{d}}\left(2 \Delta B_{\mathrm{c}} B_{\mathrm{d}}^{2}\right)^{1 / 3},
$$

where $\Delta B_{\mathrm{c}}=2 n \Delta \lambda\left(\hbar \gamma_{\mathrm{p}}\right)^{-1}$ is the maximum contact shift of the $|1\rangle-|2\rangle$ resonance in field units. Thus, the INEDOR linewidth is independent of the static field gradient. On the other hand, the spectrum intensity is inversely proportional to $|\nabla B|$.

\section{SPECTRUM NONLINEARITY OF A GAS OF TWO-LEVEL BOSONS}

In contrast to usual optical Bloch equations, Eq. (2.10) includes an essentially nonlinear term proportional to the contact shift of the resonance frequency. To observe this nonlinearity it is sufficient to have just two levels. To avoid confusion, we keep levels 1 and 3 omitting for brevity the subscripts 13 and $\mathrm{p}(\mathrm{d})$. Thus, in this section, 
$\Delta \lambda \equiv \Delta \lambda_{13}$. In addition, we set the total density constant, $n \equiv \rho_{11}+\rho_{33}=$ const. The nonlinearity is seen most clearly in the spectrum at a low enough sweep rate. In particular, if the rate $\hbar^{-1} \Delta \lambda d M_{z} / d t \sim \hbar^{-1} \Omega n \Delta \lambda$ of the variation of the transition frequency due to a change in the populations is greater than the frequency sweep rate $d \omega / d t$, the Rabi oscillations occur on the background of a relatively slow change in the magnetization along with the frequency of the light field, so that the system stays all the time near the resonance $M_{z} \Delta \lambda \approx \hbar[\omega(\rho)-\omega(0)]-n \delta \lambda$. Meanwhile, the populations periodically vary relatively quickly when the resonance conditions are fulfilled, which in turn drives the system out of resonance for a certain time until the frequency of the light field is adjusted to the new value of the transition frequency. The picture of such a reentrant resonance repeats until the transition frequency stops changing because the limiting value of the magnetization (e.g., $M_{z}=n$ ) is reached. Frequency sweep in the opposite direction is accompanied by usual behavior of the populations, since the repeated fulfillment of the resonance conditions is impossible. On the other hand, it is clear that the contact frequency shift affects the spectrum considerably if it drives the system out of the resonance conditions. This requires that the contact shift were at least comparable with the Rabi frequency (the bandwidth of the microwave generator is assumed to be sufficiently narrow; for example, the spectral width of the generator in the experiments with atomic hydrogen was $\delta f / f \lesssim 10^{-10}$ [14]) Thus, under the conditions

$$
\Omega n|\Delta \lambda| \gtrsim \hbar\left|\frac{d \omega}{d t}\right| ; n|\Delta \lambda| \gtrsim \hbar \Omega
$$

there appears a spectrum "hysteresis" (Fig. 3). If in addition $n|\Delta \lambda| \gg \hbar \Omega$, a drastic change in the spectrum upon reaching a certain critical value of the total gas density $n_{c}$ or any other parameter entering the first condition of (4.1) occurs almost abruptly (Fig. 4).

Figure 3 shows the evolution of the population of the state $|3\rangle$ during the upward and downward (indicated by the arrows) linear frequency sweep of the microwave field, according to the numeric solution of Eq. (2.10) with the parameters corresponding to the hyperfine transition $|b\rangle \rightarrow|a\rangle$ in three-dimensional atomic hydrogen, namely, at the Rabi frequency $\Omega=10 \mathrm{~s}^{-1}$, sweep rate $d \omega / d t=$ $2 \times 10^{3} \mathrm{~s}^{-2}$ and the total density $n \sim 2 \cdot 10^{18} \mathrm{~cm}^{-3}$ [14], $\Delta \lambda / \hbar=-3 \times 10^{-16} \mathrm{~cm}^{3} / \mathrm{s}$ (see Appendix). The character of the spectrum is almost insensitive to the transverse relaxation rate (see below). In the calculations, we used $\gamma=0.3 \mathrm{~s}^{-1}$. The longitudinal relaxation rate in atomic hydrogen is quite low and was therefore neglected. The total gas density corresponding to each curve is given in units of $n_{c} \approx 2.23 \times 10^{18} \mathrm{~cm}^{-3}$.

The calculated dependence of the final population of the state $|3\rangle$ on the total gas density at various sweep rates $|d \omega / d t|$ and the same values of the other parameters as in Fig. 3 is shown in Fig. 4. When the density increases from a subcritical to supercritical value, the fi-

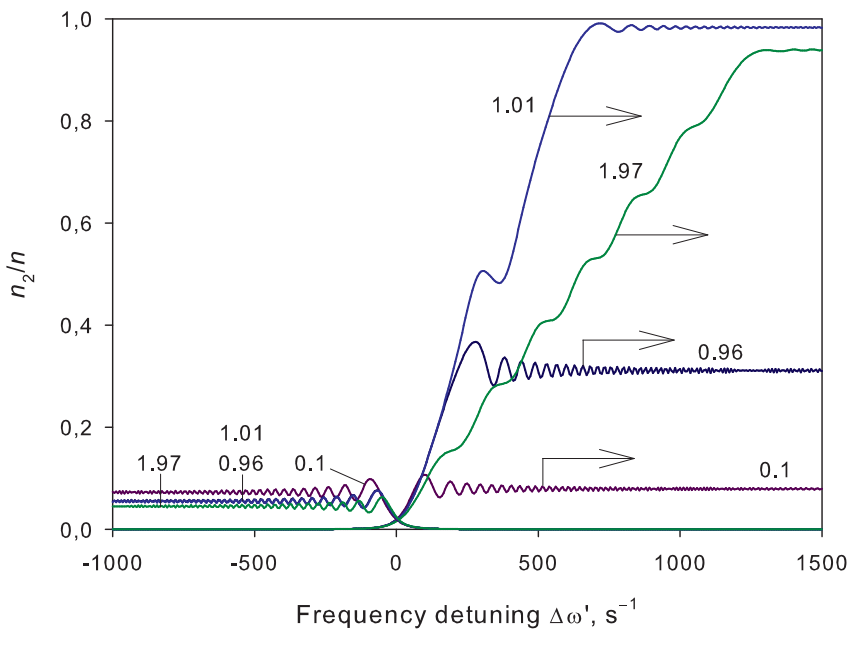

Figure 3: Evolution of the population of the state $|3\rangle$ during the linear upward (indicated by the right arrow) and downward frequency sweep of the microwave field according to the numeric solution of Eq. (2.10). The total density corresponding to each curve is marked in units of the critical density $n_{c}$. The values of the parameters correspond to the hyperfine transition $b \rightarrow a$ in three-dimensional atomic hydrogen: $\Omega=10 \mathrm{~s}^{-1}, d \omega / d t=2 \times 10^{3} \mathrm{~s}^{-2}, n_{c} \approx 2.23 \times 10^{18} \mathrm{~cm}^{-3}$, $\gamma=0.3 \mathrm{~s}^{-1}, \Gamma=0[14], \Delta \lambda / \hbar=-3 \times 10^{-16} \mathrm{~cm}^{3} / \mathrm{s}$.

nal population of the state $|3\rangle$ after sweeping through the resonance conditions sharply increases from the low value $\rho_{33} \sim \Omega^{2}|d \omega / d t|^{-1}$ given by the product of the Rabi frequency $\Omega$ and the duration $\Omega|d \omega / d t|^{-1}$ of the sweep through the resonance line, to nearly unity. A further increase in density is accompanied by a mere increase in the frequency detuning, at which the limiting population is reached. As is easily seen, this detuning is exactly the maximum possible contact frequency shift $n \Delta \lambda$. At the opposite sweep direction, the final population of the state $|3\rangle$, on the contrary, decreases with an increase in density. As one might expect, the final population is independent on the sweep direction in the low-density limit, when the contact shift vanishes. Thus, the shape of the spectrum depends on the rate and direction of the frequency sweep of the light field, as well as on the field amplitude.

As is seen in Fig. 4, the critical density $n_{c}$ (determined as an abscissa of the steepest slope) does not exactly conform to the condition $\left|\Omega n_{c} \Delta \lambda\right| \propto \hbar|d \omega / d t|$, which follows from (4.1), and rather increases superlinearly with $|d \omega / d t|$. The origin of this behavior has to be clarifies. Note only, that the period $2 \pi / \Omega$ of the Rabi oscillations at a low rate of the microwave frequency sweep (left curve in Fig. (4) becomes comparable with the duration of passing through the resonance line. Damping oscillations of the final population of the state $|3\rangle$ seen at $n>n_{c}$ presumably originate from the fact that the system finally gets out of the resonance conditions in this or that particular phase of effective Rabi oscillations (waves on the slanted parts of curves in Fig. 3 at $n>n_{c}$ ).

Equation (2.10) holds for a spatially homogeneous sys- 


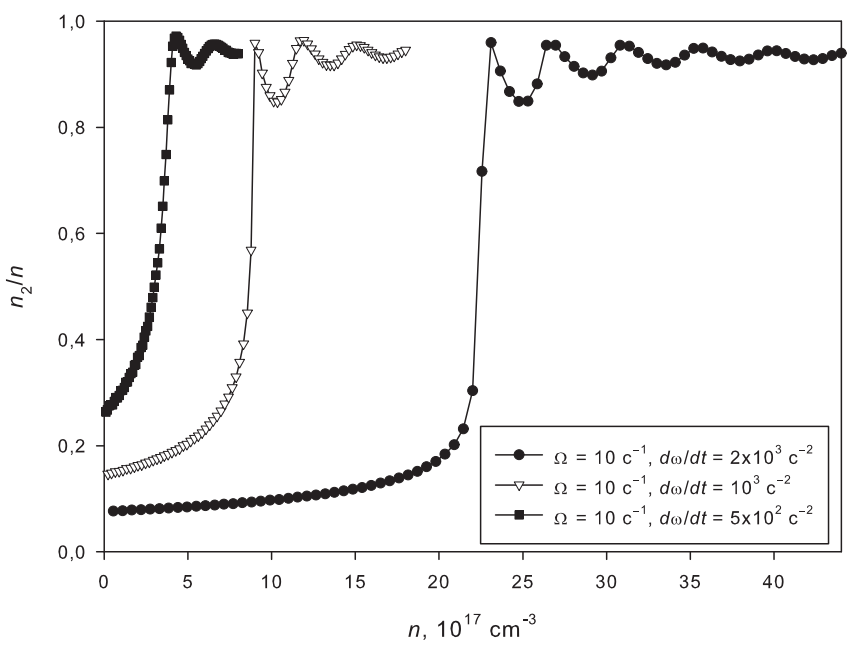

Figure 4: Calculated total-density dependence of the final population of the state $|3\rangle$ at various frequency sweep rates $|d \omega / d t|$. Other parameters are the same as in Fig. 3 .

tem. In the case of spatial inhomogeneity, there appears magnetization transport owing to exchange and dipoledipole interactions of the atomic pseudo-spins, which leads to the emergence of spin waves [10, 12]. The effect of the contact shift of the spin-wave spectrum requires separate consideration, which lies beyond the scope of the present work.

The effect described in this section can occur not only in atomic hydrogen but also in ultracold alkali vapors and a number of other systems. In any case, the particular character of interaction, which results in the dependence of the transition frequency on the population of the states involved is insignificant. The reason why the spectrum nonlinearity was not observed in the experiments on the contact shift in ${ }^{87} \mathrm{Rb}$ vapor [15] is that the second condition of (4.1) was violated. In fact, the s-wave scattering lengths of ${ }^{87} \mathrm{Rb}$ in various hyperfine states are such that the maximum differential contact shift at the density $n \sim 10^{13} \mathrm{~cm}^{-3}$ was as small as $n \Delta \lambda / h \sim 0.2 \mathrm{~Hz}$ $\left(\Delta \omega / \omega \sim 3 \cdot 10^{-11}\right)$, whereas the Rabi frequency of the two-photon transition in the pulsed microwave/RF field was about $2.5 \mathrm{kHz}$.

The dependence of the resonance field on the sample magnetization knowingly leads to a so-called ferromagnetic instability [16], e.g., of the FMR spectrum of ferromagnetic films, ESR in atomic hydrogen [17] and NMR in ${ }^{3} \mathrm{He}$ [18]. In contrast to this type of instability, whose condition is determined the transverse relaxation rate, the effect considered in this section does not depend directly on the transverse relaxation, as the contact shift in a two-level system is independent of the mutual coherence of the single-particle states [19].

We are truly grateful to S. A. Vasiliev for fruitful discussions and providing us with the data of atomic hydrogen experiments the University of Turku, Finland. This work was supported by the Human Capital Foundation, contract no. 211).

\section{Appendix A: Contact Shift in Atomic Hydrogen}

The magnitude of the contact shift of the hyperfine transition $|b\rangle \leftrightarrow|a\rangle$ in atomic hydrogen can be found as follows. The diatomic states in the basis $\left|S, m_{S} ; I, m_{I}\right\rangle$ of the total electron and nuclear spins of the pair of atoms take the form

$$
\begin{aligned}
|b b\rangle & =|1,-1 ; 1,-1\rangle \\
|a b\rangle_{+} & \equiv \frac{1}{\sqrt{2}}(|a b\rangle+|b a\rangle)= \\
& =\cos \theta|1,-1 ; 1,0\rangle-\sin \theta|1,0 ; 1,-1\rangle, \\
|a a\rangle & =\cos ^{2} \theta|1,-1 ; 1,1\rangle+\sin ^{2} \theta|1,1 ; 1,-1\rangle- \\
& -\frac{\sin 2 \theta}{2}|1,0 ; 1,0\rangle-\frac{\sin 2 \theta}{2}|0,0 ; 0,0\rangle
\end{aligned}
$$

where $\tan (2 \theta)=A\left[\left(\gamma_{e}+\gamma_{p}\right) h B\right]^{-1}, \gamma_{e}\left(\gamma_{p}\right)$ is the gyromagnetic ratio of electron (proton), $A / h=1420 \mathrm{MHz}$ is the hyperfine constant of hydrogen). That is, the states $|b b\rangle$ and $|a b\rangle_{+}$are pure electronic and nuclear triplets irrespective of the value of magnetic field. Consequently, $\lambda_{a b}^{+}=\lambda_{b b}=\frac{4 \pi \hbar^{2}}{m} a_{t}$, where $a_{t}$ is the triplet s-wave scattering length. Thus, according to Eq. (2.3), the contact shift of the transition $|b\rangle-|a\rangle$ vanishes at $\rho_{a a}=0$ in an arbitrary field. On the other had, the state $|a a\rangle$ contains the singlet component (the last term in the hight-hand side of Eq. (A3)). Consequently, $\Delta \lambda_{a b}=\lambda_{b b}+\lambda_{a a}-2 \lambda_{a b}^{+}=\frac{\pi \hbar^{2}}{m}\left(a_{s}-a_{t}\right) \sin ^{2} 2 \theta \neq 0$, $a_{t}-a_{s}=30(5) \mathrm{pm}$ [11]. In the field $B=4.5 \mathrm{~T}$, $\Delta \lambda_{a b} / \hbar=-3 \times 10^{-16} \mathrm{~cm}^{3} / \mathrm{s}$, and the frequency shift at the density $\rho_{a a}=2 \cdot 10^{18} \mathrm{~cm}^{-3}$ is about $-100 \mathrm{~Hz}$, which is two orders of magnitude greater than the Rabi frequency.

Replacing the electron spin by the nuclear spin all the aforesaid is automatically generalized to the transitions $|b\rangle \leftrightarrow|c\rangle,|a\rangle \leftrightarrow|d\rangle$ and $|c\rangle \leftrightarrow|d\rangle$, wherefrom $\Delta \lambda_{b c}=\Delta \lambda_{a d}=\Delta \lambda_{c d}=\frac{\pi \hbar^{2}}{m}\left(a_{s}-a_{t}\right) \sin ^{2} 2 \theta$. However, the populations of the states with opposite electron spins cannot be simultaneously high owing to a fast recombination of such pairs; thus, the sift of the transitions $|b\rangle \leftrightarrow|c\rangle$ and $|a\rangle \leftrightarrow|d\rangle$ is hardly detectable.
[1] B. D. Agap'ev, M. B. Gornyi, B. G. Matisov, and Yu. V. Rozhdestvenskii, Physics-Uspekhi 36 (9), 763 (1993).

[2] O. A. Kocharovskaya, Ya. I. Khanin, JETP 63, 945
(1986).

[3] A.I.Safonov, I.I.Safonova and I.S.Yasnikov, Eur. Phys. J. D 65, 279 (2011). 
[4] L. D. Landau and E. M. Lifshitz, Quantum Mechanics: Non-Relativistic Theory (Moscow: Nauka, 4th ed., 1989; Pergamon Press, Oxford, 1977, 3rd ed.), §14.

[5] G.Lindblad, Commun. Math. Phys. 48, 119 (1976).

[6] A.I.Safonov, I.I.Safonova, I.S.Yasnikov, J. Low Temp. Phys. 162, 127 (2011).

[7] K.Gibble, Phys. Rev. Lett. 103, 113202 (2009).

[8] Y.B.Band, Light and matter: electromagnetism, optics, spectroscopy and lasers, Wiley, 2006, Ch. 9.

[9] J.Ahokas, J.Jarvinen and S.Vasiliev, Phys. Rev. Lett. 98, 043004 (2007).

[10] E. P. Bashkin, JETP Lett. 33, 8 (1981).

[11] A. I. Safonov, I. I. Safonova and I. S. Yasnikov, Phys. Rev. Lett. 104 (2010) 099301.

[12] O.Vainio et al., Phys. Rev. Lett. 108, 185304 (2012) and references therein.

[13] J.Ahokas, J.Jarvinen and S.Vasiliev, J. Low Temp. Phys. 150, 577 (2007).

[14] S. A. Vasiliev, private communication.

[15] D. M. Harber, H. J. Lewandowski, J. M. McGuirk and E. Cornell, Phys. Rev. A 66 (2002) 053616.

[16] P. W. Anderson and H. Suhl, Phys. Rev. 100, 1788 (1955).

[17] S. A. Vasilyev, J. Järvinen, A. I. Safonov et al., Phys. Rev. Lett. 89, 153002 (2002).

[18] E. Stoltz, J. Tannenhauser and P.-J. Nacher, J. Low Temp. Phys. 101, 839 (1995).

[19] M. Zwierlein, Z. Hadzibabic, S. Gupta, and W. Ketterle, Phys. Rev. Lett. 91 (2003) 250404. 